\title{
SAZONALIDADE DA LAGARTA MINADORA Phyllocnistis citrella NA CITRICULTURA - UM ESTUDO DE CASO
}

\author{
Gilson Barbara1; Dagmar Aparecida de Marco Ferro² \\ ${ }^{1}$ Discente do Curso de Engenharia Agronômica, Centro Universitário - Unifunec. \\ Município: Santa Fé do Sul - Brasil.gilsoneia@hotmail.com \\ ${ }^{2}$ Graduada em Ciências Biológicas Universidade Federal de São Carlos: SP \\ Mestrado em Ecologia e Recurso Naturais: SP, Universidade Federal de São Carlos, \\ Doutorado em Genética e Evolução: Universidade Federal de São Carlos: SP.
}

Recebido em: 15/02/2020 - Aprovado em: 15/03/2020 - Publicado em: 30/03/2020 DOI: 10.18677/EnciBio_2020A21

\begin{abstract}
RESUMO
Uma das dificuldades na produção de frutas cítricas é a alta incidência de pragas. Dentre os insetos pragas destacam-se as mariposas, Phyllocnistis citrella. (Minadordas-Folhas-dos-Citros) que ocasiona danos diretos através da redução da área fotossintética com queda na produção e/ou qualidade dos frutos e danos indiretos, favorecendo a penetração da bactéria Xanthomonas citri, causadora do cancro cítrico. O objetivo deste trabalho foi estudar a incidência de Phyllocnistis citrella., sua dinâmica populacional na citricultura e a influência de fatores climáticos, em uma propriedade localizada em Santa Fé do Sul - SP. Foram analisados 1\% de 180.000 plantas da variedade Laranja Pera Rio, em 131,04 hectares. O período de observação foi de janeiro de 2017 a dezembro de 2018. Cada inspeção vistoriou três folhas por planta, com observações diárias e retorno aos talhões a cada 15 dias (48 repetições). Foram feitas análises em folhas tanto na porção interna quanto externa da planta e em toda a superfície das folhas, tanto na porção superior quanto inferior, em todo período vegetativo. Foi possível constatar uma pequena incidência da lagarta durante alguns meses no decorrer do ano, embora tivessem ocorridos picos populacionais em diferentes ocasiões de acordo com algumas particularidades. Um fator importante na identificação da flutuação populacional das lagartas é o estádio fenológico da planta, pois a praga se desenvolve em vegetações novas. A presença dos minadores nos pomares foi registrada ao final do primeiro fluxo de brotação. Os meses abril e maio, outubro e novembro se destacaram como meses que apresentaram maiores índices de infestações.
\end{abstract}

PALAVRAS-CHAVE: Citricultura. Dinâmica Populacional. Phyllocnistis citrella.

\section{SEASONALITY OF WASTE MINING Phyllocnistis citrella IN THE CITRICULTURE - CASE STUDY}

\begin{abstract}
One of the greatest difficulties in the production of citrus fruits is the high incidence of pests. Amongst the plague insects the moths Phyllocnistis citrella ( Citrus Leaf Miner) stand out, causing direct damage by reducing the photosynthetic area, decreasing productivity and/or quality of the fruits as well as indirect damage causing the
\end{abstract}


penetration of the Xanthomonas citri bacteria that causes citric cancer. Our goal was to study the occurrence of CLM and its population dynamics in citriculture, as well as the influence of climate factors in a farm in the city of Santa Fé do Sul - SP. $1 \%$ of 180.000 plants of the variety Laranja Pera Rio were analyzed between January 2017 and December 2018. In each inspection, three leaves per plant were analyzed with daily observations, going back to the plantation every 15 days, with a total of 48 repetitions. The sample collections were done inside and outside the leaves and in all their surface. The presence of CLM was observed with a small incidence throughout the year, although there were population peaks in different occasions according to particularities and biology of the species observed. An important factor when identifying the population flow of the CLM in the citrus is related to the phenological stage of the plant, meaning the plague develops in young vegetation. The presence of the moths was registered at the end of the first cycle of the budding flow. April and May, October and November stood out as months with the greatest levels of infestation.

KEYWORDS: Citriculture. Population dynamics. Phyllocnistis citrella.

\section{INTRODUÇÃO}

O Brasil é um dos principais países produtores de frutas cítricas do mundo. Mesmo com essa colocação no ranking da produção de citrus, uma das grandes dificuldades para a produção é a alta incidência de pragas na citricultura (KUMAR et al., 2017; NEGRI; et al., 2018).

A cultura de citrus, durante os últimos 15 anos, obteve grande avanço na tecnologia de controle de pragas. Mesmo assim uma das principais dificuldade para a produção continua sendo a alta incidência das pragas consideradas primárias para a cultura ( ANDRADE, 2016; PAVARINI, 2016; AZEVEDO; MORELI, 2018; CRISTOFANI, 2019).

Os insetos-praga se manifestam desde a formação das mudas até a implantação e condução do pomar e podem comprometer o desenvolvimento e a produtividade das plantas ou mesmo inviabilizá-la economicamente. Dentre os insetos pragas de extrema importância para a citricultura destacam-se as mariposas, Phyllocnistis citrella. Por ser importante, o estudo da dinâmica populacional desse inseto praga tem sido realizada em diferentes variedades de citrus, nos principais países produtores do mundo ( LEGASPI et al., 2000; PUTRUELLE; PETIT, 2000; PATEL, 2001; AMALIN, 2002). O minador-das-folhas-dos-citros, $P$. citrella, ocasiona danos diretos na planta através da redução da área fotossintética com comprometimento da produção primária e consequente queda na produção e/ou qualidade dos frutos (KNAPP et al., 1993; SARADA et al., 2014; PAIVA; YAMAMOTO, 2014; FLORES; VEITÍA, 2016). Segundo Moreira (1975), esse minador também causa danos indiretos aos citrus, pois favorece a penetração da bactéria Xanthomonas citri causadora do cancro cítrico que, em vista da sua gravidade, exige a queima das plantas para evitar o alastramento da doença,

O cancro cítrico é uma das principais doenças do citrus sendo conhecida mundialmente como a "doença a ser batido", apresentando um potencial devastador da produção de citrus, especialmente aquelas cultivadas em áreas onde são registradas, simultaneamente, chuva em abundância e temperaturas elevadas (PRUVOST et al.,1997). De acordo com Barbosa et al. (2001), o controle do cancro cítrico no estado de São Paulo e também em outros estados, baseia-se em medidas de exclusão e erradicação de plantas dos pomares quando afetadas pela doença. Todavia, áreas com a presença da lagarta minadora do citros (LMC) têm relação 
direta com o aumento na incidência do cancro cítrico, sendo essencial a aplicação de medidas de controle, a fim de diminuir a incidência da doença nos pomares (GRAVENA, 1994; LOURENÇÃO; MULLER, 1994). O Brasil por ser um pais em condições climáticas tropicais, apresenta grande número de incidência de pragas nas culturas. A lagarta minadora se destaca como praga principal na cultura do citrus, sendo encontrada em grande parte dos pomares produtivos, (ANDRADE et al., 2014).

Sabendo-se que as condições ambientais exercem influência direta sobre a dinâmica populacional dos insetos, o objetivo desse trabalho, caracterizado como um estudo de caso, foi o levantamento do índice populacional da lagarta minadora da cultura do citrus em uma propriedade no município de Santa Fé do Sul, localizado no noroeste paulista.

\section{MATERIAL E METODOS}

A pesquisa foi desenvolvida na propriedade Fazenda Recanto da Onça no município de Santa Fé do Sul/ SP localizada na Rodovia dos Barrageiros, km $96 \mathrm{~S} / \mathrm{N}$ Bairro do Peba, Latitude $20^{\circ} 29^{\prime}$ 09" sul e Longitude $51^{\circ} 37^{\prime} 45^{\prime \prime}$ oeste, estando a uma altitude de 370 metros. Foram analisados $1 \%$ de 180.000 plantas da variedade Laranja Pera Rio, abrangendo uma área experimental total de 131,04 hectares (FIGURA 1).

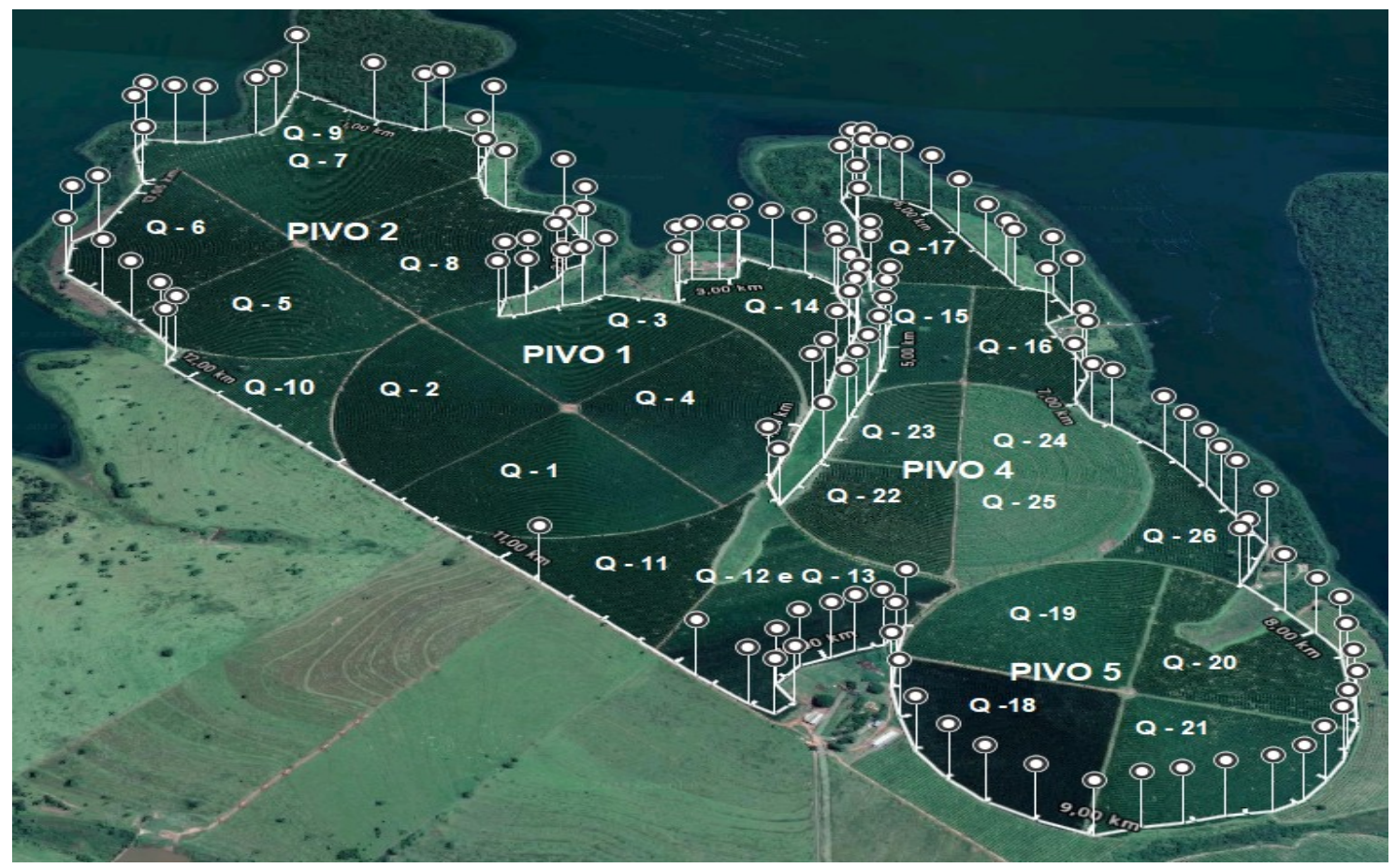

FIGURA 1: Área total de Produção Avaliada.

Fonte: GOOGLE MAPS, (2019)

A variedade observada foi a laranja pera rio com plantio realizado em 2008, em espaçamento de sete metros por ruas e 2,5 metros entre plantas. As amostras avaliadas foram divididas em talhões identificados com numeração de um a 26 . A inspeção ocorreu diariamente por um período de 24 meses nos anos de 2017 e 2018, (QUADRO 1). O experimento teve inicio com as observações no dia 10 de janeiro de 2017 e seu término ocorreu no dia 21 de Dezembro de 2018. 
QUADRO 1: Monitoramento diário de Incidência de lagarta minadora do citros em 2017 e 2018

\begin{tabular}{|c|c|c|c|c|c|c|c|c|c|c|c|c|c|c|c|c|c|c|c|c|c|c|c|c|}
\hline DIA & JAN & FEV & MAR & ABR & MAI & JUN & JUL & AGO & SET & OUT & Nov & DEZ & JAN & FEV & MAR & ABR & MAI & JUN & JUL & AGO & SET & OUT & Nov & DEZ \\
\hline 1 & & Q-7 & Q-3 & $\mathrm{s}$ & Q-8 & Q-8 & S & Q-21 & Q-7 & D & -21 & Q-7 & -19 & Q-20 & Q-23 & D & -25 & Q-14 & D & Q-22 & $s$ & Q-22 & Q-11 & s \\
\hline 2 & & Q-3 & Q-9 & $\mathrm{D}$ & 7 & Q-15 & D & 3 & $s$ & Q-12 & Q-15 & $\mathrm{s}$ & 20 & Q-20 & Q-14 & Q-5 & 10 & $\mathrm{~s}$ & Q-13 & -5 & 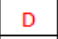 & $2-24$ & Q-26 & D \\
\hline 3 & & Q-11 & Q-13 & Q-23 & Q-24 & $S$ & Q-7 & Q-10 & D & Q-5 & 7 & D & Q-6 & $S$ & $\mathrm{~s}$ & 9 & 7 & D & 21 & Q-9 & Q-10 & -2 & $\mathrm{~s}$ & Q-18 \\
\hline 4 & & $\mathrm{~s}$ & $S$ & Q-6 & 0 & D & -1 & Q-15 & Q-2 & Q-2 & $S$ & Q-14 & $Q-22$ & 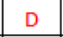 & 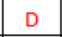 & Q-22 & -4 & Q-20 & $0<0$ & 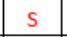 & Q-21 & Q-26 & D & $2-18$ \\
\hline 5 & & 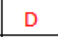 & 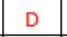 & Q-9 & Q-10 & Q-21 & -4 & s & Q-9 & Q-8 & D & 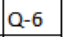 & $2-11$ & Q-26 & Q-6 & -1 & $S$ & -19 & Q-24 & $\Delta$ & Q-12 & $Q-26$ & $Q-1$ & Q-13 \\
\hline 6 & & Q-24 & Q-7 & Q-15 & $S$ & -18 & -4 & 政 & Q-20 & Q-21 & Q-4 & Q-23 & $S$ & Q-5 & Q-22 & Q-22 & 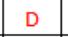 & Q-9 & Q-22 & Q-3 & 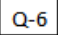 & S & Q-13 & Q-4 \\
\hline 7 & S & -17 & Q-15 & Q-6 & D & Q-9 & Q-12 & Q-23 & Q-2 & $S$ & Q-14 & Q-17 & D & Q-25 & Q-10 & 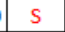 & Q-11 & Q-24 & 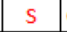 & Q-17 & Q-18 & D & Q-14 & Q-15 \\
\hline 8 & D & 12 & Q & $S$ & 10 & 2 & $S$ & Q-17 & Q-18 & D & Q-7 & Q-12 & Q-8 & Q-5 & & 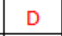 & -5 & Q-11 & 政 & 2. & $S$ & Q-9 & Q-1 & $S$ \\
\hline 9 & & 23 & Q-17 & D & -10 & 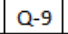 & 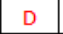 & Q-16 & 5 & Q-18 & Q-22 & S & Q-22 & Q-12 & Q-4 & Q-11 & Q-10 & $\mathrm{S}$ & Q-21 & Q-26 & D & Q-22 & $Q-2$ & D \\
\hline 10 & -21 & Q-20 & Q-22 & Q-12 & Q-17 & 3 & Q-13 & Q-12 & D & Q-21 & Q-3 & D & {$[-13$} & S & 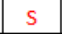 & Q-17 & Q -1 & D & Q-16 & Q-22 & Q-11 & Q-6 & S & Q-16 \\
\hline 11 & Q-19 & 3 & 3 & Q-25 & Q-10 & D & Q-19 & Q-26 & Q-14 & Q-25 & 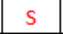 & Q-4 & $Q=0$ & D & D & Q-11 & Q-12 & Q-4 & Q-12 & 3 & Q-5 & $Q-19$ & D & Q-2 \\
\hline 12 & -4 & 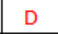 & D & Q-22 & Q-25 & Q-17 & Q-25 & 3 & Q-7 & Q-26 & D & Q-17 & Q-22 & Q-20 & Q-22 & Q-13 & 3 & Q-23 & Q-16 & 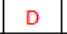 & Q-3 & Q-24 & Q-15 & Q-12 \\
\hline 13 & Q-5 & -12 & Q-20 & Q-26 & 3 & Q-14 & $2-2$ & 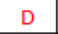 & Q-24 & Q-12 & Q-1 & Q-8 & $\mathrm{S}$ & Q-1 & Q-5 & Q-17 & U & Q-14 & Q-22 & Q-17 & Q-14 & 3 & Q-11 & Q-2 \\
\hline 14 & $S$ & $Q-12$ & Q-10 & Q-20 & D & -12 & Q-21 & Q-12 & Q-21 & $S$ & Q-6 & Q-15 & D & Q-15 & Q-15 & 3 & Q-10 & Q-25 & 3 & $\mathrm{Q}-3$ & Q-3 & D & $2-4$ & Q-4 \\
\hline 15 & D & -4 & Q-22 & $s$ & Q-21 & Q-11 & $\mathrm{s}$ & Q-20 & Q-5 & D & Q-20 & Q-1 & Q-21 & $Q-3$ & $1-6$ & D & Q-24 & Q-4 & U & Q-20 & $S$ & $2-10$ & Q-7 & $S$ \\
\hline 16 & Q-12 & Q-16 & Q-1 & D & Q-9 & Q-9 & D & Q-8 & $S$ & Q-17 & Q-4 & $\mathrm{s}$ & $Q-2$ & $Q-5$ & Q-3 & $Q-5$ & Q-14 & $S$ & Q-19 & Q-16 & D & $Q-3$ & Q-6 & D \\
\hline 17 & Q-26 & Q-19 & Q-15 & Q-21 & Q-26 & $\mathrm{s}$ & Q-9 & Q-5 & D & Q-5 & Q-10 & $D$ & Q-13 & S & $\mathrm{s}$ & $Q-8$ & Q-16 & $\mathrm{D}$ & Q-19 & Q-4 & Q-5 & Q-15 & $S$ & $Q-17$ \\
\hline 18 & Q-22 & S & $\mathrm{s}$ & Q-21 & Q-20 & D & Q-3 & Q-16 & Q-26 & Q-17 & S & Q-9 & Q-13 & D & $\mathrm{D}$ & Q-3 & Q-5 & Q-24 & Q-12 & s & Q-8 & $Q-2$ & D & Q-11 \\
\hline 19 & Q-5 & D & D & Q-25 & Q-10 & Q-13 & Q-5 & $S$ & Q-1 & Q-22 & D & Q-13 & Q-17 & Q-18 & Q-13 & $Q-6$ & $S$ & Q-24 & Q-5 & D & Q-1 & Q-16 & Q-5 & Q-13 \\
\hline 20 & Q-20 & Q-15 & Q-15 & Q-24 & $\mathrm{S}$ & Q-9 & Q-19 & D & Q-23 & Q-24 & Q-1 & Q-5 & $\mathrm{s}$ & Q-24 & Q-9 & Q-20 & D & Q-19 & Q-10 & Q-9 & Q-16 & S & Q-6 & Q-18 \\
\hline 21 & $\mathrm{~s}$ & Q-24 & Q-17 & Q-11 & D & Q-16 & Q-6 & Q-23 & Q-19 & $\mathrm{s}$ & Q-13 & Q-7 & D & Q-4 & $Q-2$ & S & Q-18 & Q-12 & $\mathrm{s}$ & Q-14 & Q-9 & D & Q-23 & Q-20 \\
\hline 22 & $\mathrm{D}$ & Q-8 & Q-10 & $\mathrm{s}$ & Q-25 & Q-10 & S & Q-19 & Q-24 & D & Q-6 & Q-5 & Q-23 & $Q-5$ & Q-18 & D & Q-22 & Q-3 & D & Q-6 & $\mathrm{s}$ & Q-14 & Q-6 & \\
\hline 23 & Q-9 & Q-14 & Q-8 & $\mathrm{D}$ & Q-25 & Q-5 & D & Q-17 & $\mathrm{s}$ & Q-7 & Q-17 & s & Q-6 & Q-14 & Q-6 & $Q-7$ & Q-13 & $\mathrm{s}$ & Q-24 & Q-18 & D & Q-22 & Q-10 & \\
\hline 24 & Q-19 & Q-20 & Q-10 & Q-2 & Q-14 & $S$ & Q-10 & Q-22 & D & Q-15 & Q-24 & $\mathrm{D}$ & 14 & 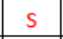 & $\mathrm{s}$ & Q-5 & Q-1 & D & $\mathrm{Q}-2$ & Q-4 & Q-23 & $2-9$ & $S$ & \\
\hline 25 & Q-6 & $S$ & $\mathrm{~s}$ & Q-21 & Q-25 & D & Q-19 & Q-10 & Q-6 & Q-26 & 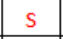 & $\mathrm{F}$ & Q-14 & 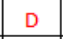 & $\mathrm{D}$ & Q-5 & Q-17 & Q-19 & Q-22 & $S$ & -4 & 2-1 & D & \\
\hline 26 & Q-13 & 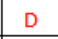 & 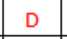 & Q-2 & Q-1 & Q-12 & Q-14 & $S$ & Q-21 & Q-6 & D & Q-21 & Q-14 & Q-7 & Q-4 & Q-14 & $S$ & Q-6 & 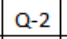 & 8 & Q-13 & Q-20 & Q-24 & \\
\hline 27 & Q-23 & Q-16 & Q-17 & Q-6 & $\mathrm{s}$ & Q-16 & 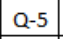 & D & Q-21 & Q-26 & Q-13 & Q-20 & 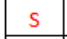 & Q-22 & Q-19 & Q-4 & D & -18 & Q-18 & Q-10 & Q-25 & $S$ & $2-6$ & \\
\hline 28 & s & 25 & Q-16 & Q-5 & 政 & Q-4 & Q-19 & $1-8$ & Q-15 & $S$ & 2-25 & Q-19 & D & Q-23 & & & Q-7 & Q-3 & 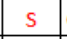 & Q-15 & Q-12 & D & Q-11 & \\
\hline 29 & 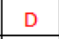 & & Q-20 & $\mathrm{s}$ & Q-9 & Q-14 & $S$ & Q-11 & Q-10 & 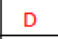 & Q-15 & Q-17 & 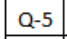 & & & & Q-25 & Q-5 & 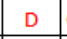 & Q-18 & $S$ & $2-14$ & $2-2$ & \\
\hline 30 & $\alpha-10$ & & 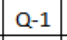 & D & 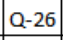 & 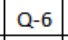 & 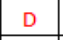 & Q-26 & $\mathrm{s}$ & Q-22 & 0 & $s$ & & & Q-18 & Q-16 & 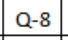 & $\mathrm{s}$ & Q-10 & Q-22 & D & $2-23$ & $2-3$ & \\
\hline 31 & Q-15 & & Q-14 & & Q-18 & & Q-16 & Q-26 & & $4=5$ & & U & $42-24$ & & $S$ & & Q-1 & & a & $Q<-4$ & & Q-12 & & \\
\hline
\end{tabular}

\section{Fonte: Próprios Autores}

No levantamento foi analisado $1 \%$ das plantas do pomar, com retorno aos talhões no máximo a cada 20 dias, totalizando 48 repetições de observações por talhão. Para observação foi utilizado uma lente $10 x$ mais e um caderno para anotações. Com relação ao minador do citros, as coletas das amostras foram realizadas em folhas localizadas na porção interna e externa da planta, isto é, mais distante do caule e também em todas as regiões das folhas, tanto na parte superior quanto inferior, em todo período vegetativo da planta. Durante a avaliação a leitura foi manuscrita na ficha do técnico (pragueiro) de acordo com o dano provocado em cada quadra. Todos os levantamentos e dados estatísticos foram embasados em considerações da metodogia da empresa de pesquisa científica FUNDECITRUS Fundo de Defesa da Citricultura. 


\section{RESULTADOS E DISCUSSÃO}

As observações no decorrer do experimento, ao longo de dois anos, mostraram a presença da lagarta minadora durante alguns meses do ano, destacando alguns picos populacionais, conforme pode ser destacado nos Graficos 1 e 2 .

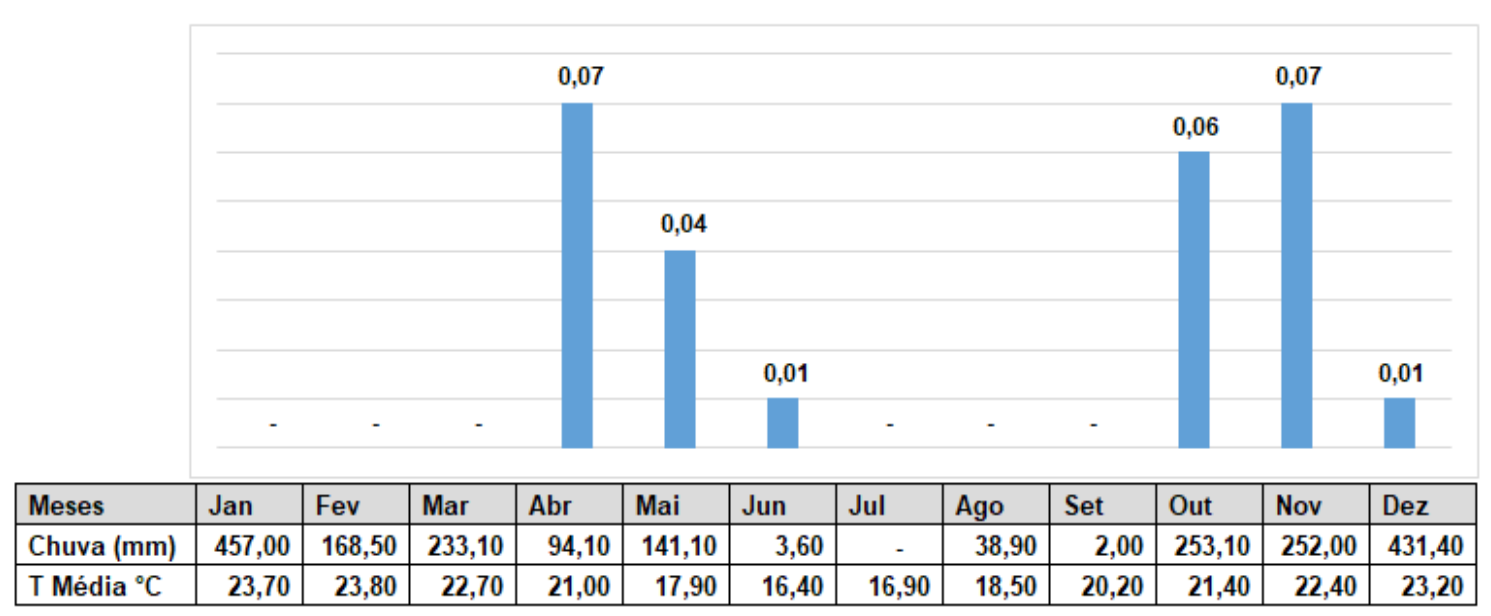

GRÁFICO 1: Observação da presença da lagarta minadora no ano de 2017 Fonte: Próprios Autores

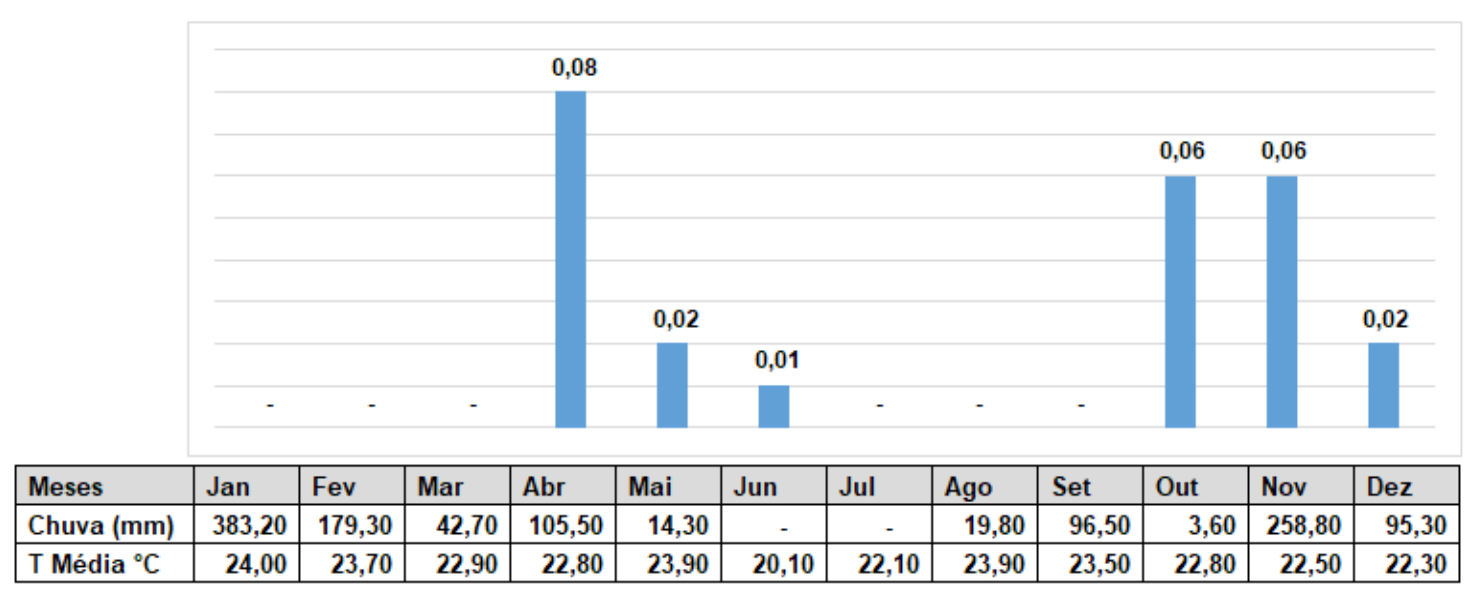

GRÁFICO 2: Observação da presença da lagarta minadora no ano de 2018 Fonte: Próprios Autores

Através da análise dos gráficos, pode-se observar que os meses de Abril, Maio, Junho, Outubro, Novembro e Dezembro, tanto em 2017 como em 2018, foram meses em que ocorreram aumento no número de lagartas nas plantações. Com relação a temperatura, esta não apresentou diferença significativa em relação aos meses restantes do ano, seja em 2017 ou 2018. Mesmo o mês de Junho de 2017 apresentando a menor temperatura, quando comparado aos dois anos, não houve variação do número de lagartas.

Quanto a umidade relativa do ar, em 2017 pôde ser observado o índice pluviométrico apresentou variação, sendo o mês de Julho o de menor índice $(3,60$ $\mathrm{mm})$. Coincidentemente, foi o mês com menor número de lagartas. Entretanto, Dezembro apresentou uma quantidade de chuva maior que o restante dos meses, o 
que é característico para essa época do ano, mas manteve uma porcentagem de lagartas semelhante a Junho que apresentou o menor índice. Em 2018, a quantidade de chuva foi bem inferior ao ano de 2017. Entretanto as porcentagens de lagartas permaneceram muito próximas.

Durante as inspeções, observando as programações internas da propriedade relativo aos manejos fitossanitários, foi possível perceber que os níveis populacionais da LMC mantiveram-se baixos durante todo o período de avaliação. De acordo com Parra (2002), os resultados obtidos em novas pesquisas mostram que a população do minador-dos-citros, Phyllocnistis citrella, pode ser reduzida pela ação de parasitoides ou através de controle químico. A região de Limeira (SP), uma das cinco maiores regiões citrícolas do Estado de São Paulo, apresenta as condições básicas necessárias para o desenvolvimento da LMC, ou seja, umidade relativa do ar superior a $60 \%$ e temperaturas médias acima de $25^{\circ} \mathrm{C}$, durante os meses de setembro a abril, período no qual se concentram os maiores fluxos de brotações novas (CHAGAS, 1999). Entretanto, mantem o patamar de produtividade realizando um controle através de inspeção periódica dos pomares.

A inspeção periódica é de fundamental importância, pois permite detectar a presença das pragas e o grau de infestação. A decisão deve ser tomada após a inspeção e a avaliação da presença dos insetos pragas e dos inimigos naturais. $O$ Manejo Integrado de Pragas - MIP, é uma técnica que mantém as pragas sempre abaixo do nível de dano econômico e ambiental. O controle destas pragas pode ser químico ou biológico, sendo que todas as técnicas empregadas no MIP são validadas por pesquisas e experiências de campo (CARVALHO; BARCELLOS, 2012).

Com relação a lagarta minadora, a presença de $10 \%$ ou mais de infestação na área de produção são considerados índice de dano econômico pela agressividade dos danos causados e por permitir a entrada de outros organismos que provocam doenças como o cancro cítrico. Durante todo o período de avaliação foram realizadas leituras diárias das médias de temperatura máxima e mínima, na propriedade, além de acompanhar diariamente o histórico pluviométrico da região local.

Outro fator importante para identificar a flutuação populacional das lagartas minadoras dos citros está relacionado com o estádio fenológico da planta, quando o maior índice de infestação acontece na vegetação mais nova, maior é o dano econômico diminuindo o espaço foliar de realização de fotossíntese além de necrosar as folhas e aberturas para os patógenos (FIGURA 2).

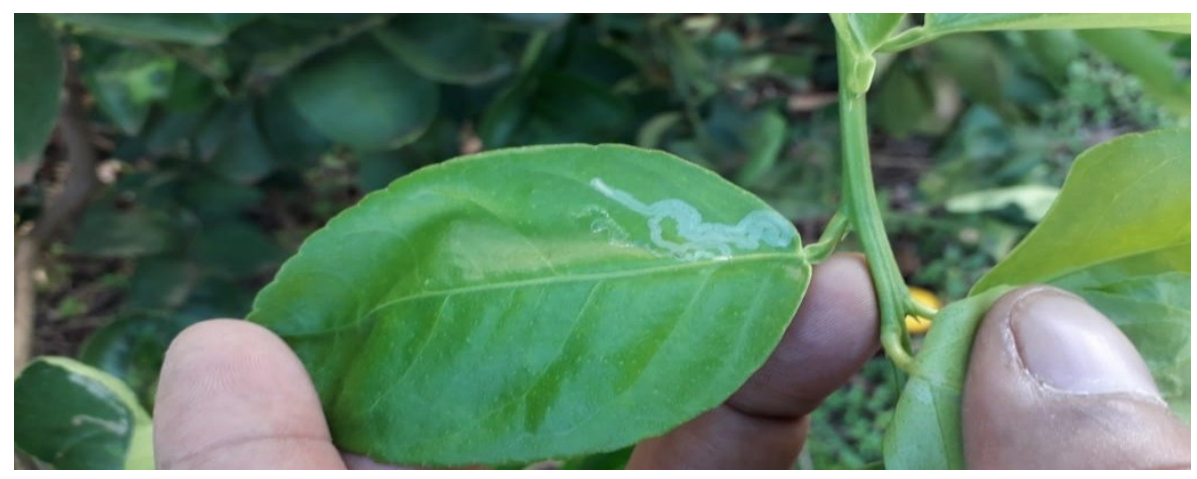

FIGURA 2: Lagarta minadora do Citros.

Fonte: Próprios Autores 
A minadora dos citros, prefere as brotações iniciais, atacando normalmente as folhas novas, podendo em alta infestação, ser notada em ramos novos e frutos (NEGRI; MACHADO; SANTOS, 2018). A presença dos minadores nos pomares pode ser registrada, em geral, ao final do primeiro fluxo de brotação. A vegetação e a elevação da temperatura são as condições identificadas como as mais favoráveis ao desenvolvimento das lagartas minadoras (JAHNKE, 2008).

Desta forma o manejo integrado de pragas (MIP) deve ser realizado de forma mais técnica e criteriosa, podendo garantir a sustentabilidade do setor citrícola, permitindo expressivos ganhos econômicos, sem causar maiores problemas ao ambiente e ao próprio homem (YAMANOTO, 2008).

\section{CONCLUSÃO}

Com os dados obtidos com a pesquisa envolvendo a lagarta minadora, concluímos que é preciso maior atenção com a produção, principalmente durante a primavera onde observamos as primeiras brotações, pois as lagartas preferem folhas jovens. Os meses de abril, maio, junho, outubro; novembro e dezembro se destacaram como os meses que apresentaram maior incidência. A forma de minimizar a ação da lagarta minadora é extremamente importante, pois existe toda uma preocupação por causa do favorecimento ao abrir espaços para a entrada de patógenos sendo um deles o causador da doença do cancro cítrico. É extremamente importante o controle com técnicas biológicas utilizando parasitoides que já estão sendo empregados e também o controle químico com defensivos agrícolas para a eliminação das lagartas.

\section{REFERÊNCIAS}

AMALIN, D.M. Natural mortality factors acting on citrus leafminer, Phyllocnistis citrella, in lime orchards in South Florida. BioControl, v.47, p.327-347, 2002.

ANDRADE, D. J.; FERREIRA, M. C.; MARTINELI, M. N. Aspectos de Fitossanidade em Citros. Jaboticabal: SP, UNESP. p. 23.2014.

ANDRADE, F. P. Confundimento sexual de Phyllocnistis citrella Stainton, 1856 (Lepidoptera: Gracillariidae) com o uso de feromônio sintético. Araraquara: SP. Fundecitrus, p. 31, 2016.

AZEVEDO, F. A.; MORELLI, M. Dia do Huanglongbing: alerta mantido. Cordeiropolis: SP. Centro de Citricultura, 2018.

BARBOSA, J.C., GIMENES-FERNANDES, N., MASSARI, C.A., AYRES, A.J. 2001. Incidência e distribuição de cancro cítrico em pomares comerciais do Estado de São Paulo e Sul do Triângulo Mineiro. Summa Phytopathologica 27:30-35.

CARVALHO, N. L.; BARCELLOS, A. L. Adoção do manejo integrado de pragas baseado na percepção e educação ambiental. Revista Eletrônica em Gestão, Educação e Tecnologia Ambiental, Santa Maria, v. 5, n.5, p.749-766, 2012.

CHAGAS, M. C. M. das. Phyllocnistis citrella Stainton, 1856 (Lepidoptera: Gracilariidae): bioecologia e relação com o cancro cítrico. 1999. 67 p. Tese (Doutorado em Ciências) - Escola Superior de Agronomia Luís de Queiroz, Universidade de São Paulo, Piracicaba. 
CRISTOFANI, M. Acaro da Leprose no Citros, ainda saberemos. Cordeiropolis:SP, Centro de Citricultura, 2019.

FLORES, D. D. C.; VEITÍA, M. Evaluación de cinco insecticidas para el control de las poblaciones de Phyllocnistis citrella (Lepidoptera: Gracillariidae) en cultivos de toronja en Cuba. Revista Cubana de Ciencias Biológicas, v. 4, n. 3, p. 43-49, 2016.

GOOGLE MAPS, Disponivel em: < https://www.google.com.br/maps/place/Santa+F \%C3\%A9+do+Sul,+SP,+15775-000/@-20.2885351,-50.9685484,2450m/data=!3m1! 1e3!4m5!3m4!1s0x9499c14d8e677679:0xca204176452cd67d!8m2!3d-20.2114344! 4d-50.9271748>. Acesso: Maio, 2019.

GRAVENA, S. ;"Minadora das folhas dos citros”, a mais nova ameaça da citricultura brasileiral. Laranja 15:397-404.1994.

JAHNKE, S. M. Interações entre insetos-praga e seus inimigos naturais em pomares orgânicos de citros. Pelotas: RS. Programa de Pós-Graduação em Fitotecnia da Universidade Federal do Rio Grande do Sul, p. 16, 2008.

KNAPP, J.L.; PENA, J.E.; STANSLY, P.A; HEPPNER, J.B.; YANG, Y. Citrus leafminer, a new pest of citrus in Forida. Citrus Industry. p.42-43, oct., 1993.

KUMAR, K.; ARORA, P. K.; GILL, M. I. S. Breeding Avenues in Fruit Crops for Imparting Resistance Against Insect Pests. In: Breeding Insect Resistant, Crops for Sustainable Agriculture. Singapore: Springer, $2017 . \quad$ p.289-322. https://doi.org/10.1007/978-981-10-6056-4_10.

LEGASPI, J.C. et al. The citrus leafminer (Lepidoptera: Gracillariidae) in south Texas: Incidence and parasitism. Florida Entomologist, v.82, n.2, p.305-316, 2000.

LOURENÇÃO, A.L., MULLER, G.W. 1994. Minador das folhas dos Citros: praga exótica potencialmente importante para a citricultura brasileira. Laranja 15:405-412.

MOREIRA, S. Cancro cítrico: ameaça à citricultura brasileira. Revista de Agricultura, v. 50, n.1-2, p. 79-84, 1975.

NEGRI, J. D.; MACHADO, M. A.; SANTOS, V. M. Mudanças climáticas e produção de citros. Cordeiropolis: SP. Centro de Citricultura, 2018.

PAIVA, P. E. B.; YAMAMOTO, P. T. Citrus caterpillars, with an emphasis on Helicoverpa armigera: a brief review. Citrus Research and Technology, v. 35, n. 1, p. 11-17, 2014. https://doi.org/10.5935/2236-3122.20140002.

PATEL, G.P.; PATEL, J.R. Population dynamics of on citrus in middle Gujarat. Indian Journal of Entomology, v.63, p.41-48, 2001.

PARRA, J. R. P. Controle Biológico Das Pragas De Citros. Bebedouro: SP. Editora Novos Talentos, p.32, 2002. 
PAVARINI, M. P. Flutuação populacional de Helicoverpa armigera (Hübner) (Lepidoptera: Noctuidae) e descrição das injúrias em citros. Araraquara: SP, Fundecitrus, p.22, 2016.

PUTRUELE, M.T.G.; PETIT MARTY, N. Dinámica poblacional del Minador de la hoja de los Cítricos en el NE de Entre Ríos. Revista El Horizonte del Productor, v.11, p.30-33, 2000.

PRUVOST, O., VERNIĖRE, C., HARTUNG, J., GOTTWALD, T.R., QUETELARD, H. Towards an improvement of citrus craker control in reunion island. Fruits 52(6):375382.1997.

SARADA, G.; GOPAL, K.; GOURI SANKAR, T., MUKUNDA LAKSHMI, L., GOPI, V., NAGALAKSHMI, T., RAMANA, K. T. V. Citrus Leaf Miner (Phyllocnistis citrella Stainton, Lepidptera: Gracillariidae): Biolology and Management: A Review. Research and Reviews: Journal of Agriculture and Allied Sciences, v.3, n.3, p.3948, 2014.

YAMANOTO, P. T. Manejo integrado de pragas dos citros. Piracicaba: 2008. $336 \mathrm{p}$. 